\title{
Construcción reflexiva de enunciados epistemológicos en el laboratorio de bioquímica de la UPEL-IPC: Una experiencia exploratoria
}

\author{
Reflective construction of epistemological statements in the UPEL-IPC \\ biochemistry laboratory: an exploratory experience \\ Construção reflexiva de declarações epistemológicas no laboratório de \\ bioquímica UPEL-IPC: Uma experiência exploratória
}

ARTÍCULO ORIGINAL

\author{
Julia L. Flores-Espejo \\ jflorespejo@hotmail.com \\ ORCID: 0000-0001-6286-4021
}

Universidad Pedagógica Experimental Libertador (UPEL), Caracas, Venezuela

Recibido febrero 2020 | Revisado marzo 2020 | Publicado en julio 2020

\section{RESUMEN}

Esta experiencia exploratoria permitió caracterizar el proceso de construcción reflexiva de enunciados epistemológicos sobre la ciencia de ocho docentes en formación del área de Química durante el desarrollo de cinco trabajos prácticos de laboratorio (TPL) de Bioquímica de la UPEL/IPC contextualizados disciplinariamente bajo un enfoque constructivista, usando el diagrama $\mathrm{V}$ por pares. Los enunciados epistemológicos de construcción propia se tomaron de la sección de filosofía de este diagrama en su versión final como informe de laboratorio, después de ser mejorados reflexivamente en las diferentes fases del laboratorio. El análisis de contenido reveló cuatro tipos de enunciados emergentes, los conceptos utilizados para su construcción y una cierta tendencia epistemológica general. La observación participante identificó dificultades en la experiencia exploratoria, permitiendo una reflexión docente continua con fines didácticos. La disposición reflexiva de los estudiantes se revela en los enunciados epistemológicos construidos. Los resultados fortalecerán esta línea de investigación.

Palabras clave: diagrama; enunciados epistemológicos: naturaleza de la ciencia:

\section{ABSTRACT}

This exploratory experience allowed to caharacterize the process of reflective construction of epistemological statements about science of eight teachers in training the Chemistry area during the development of five disciplined contextualized practical laboratory work (TPL) of Biochemistry of the UPEL/IPC under a constructivist approach using the $\mathrm{V}$ diagram by pair team. The self-made epistemological statements were taken from the philosophy section of this diagram in its final version as a laboratory report, after being reflexively refined in the different phases of the laboratory. The content analysis revealed four types of emergent statements, the concepts used in this construction and a certain general epistemological tendency. The participant observation identified difficulties in the exploratory experience, allowing a continues teaching reflection for didactic purpose. The reflective disposition of the students is revealed in the constructed epistemological statements. The results will strengthen this research line.

Key Words: V diagram; epistemological statements; nature of science; laboratory 


\section{RESUMO}

Essa experiência exploratória permitiu caracterizar ou refletir o processo de construção de enunciados epistemológicos sobre ciências por professores em formação na área de Química durante ou desenvolvimento de cinco trabalhos práticos de laboratório (TPL) em Bioquímica na UPEL / IPC, disciplinados contextualizados na abordagem., usando ou vinculado o diagrama V. As declarações epistemológicas feitas por nós pela primeira vez são retiradas da seção de filosofia deste diagrama em sua versão final como um relatório de laboratório, refinado de forma reflexiva em diferentes fases do laboratório. Uma análise de conteúdo revelou quatro tipos de afirmações emergentes, os conceitos utilizados para sua construção e uma certa tendência epistemológica geral. Uma observação participante identificou dificuldades na experiência exploratória, permitindo a reflexão contínua do professor para fins didáticos. Uma disposição reflexiva de dois estudos e nos revelou construiu afirmações epistemológicas. Os resultados fortalecerão essa linha de pesquisa.

Palavras-chave: Diagrama; declarações epistemológicas; natureza da ciência; laboratório

\section{INTRODUCCIÓN}

Actualmente se considera que la enseñanza del laboratorio de ciencias que se ha venido desarrollando tradicionalmente mediante trabajos prácticos altamente estructurados, tipo "recetas de cocina", distorsiona la naturaleza esencial del proceso investigativo y contribuye a una concepción deformada de la ciencia, en general. En consecuencia se ha recomendado que los estudiantes participen en un ambiente de aprendizaje que refleje los procedimientos de la ciencia, es decir, la actividad investigativa, como contexto apropiado para posibilitar una comprensión adecuada de la ciencia (Díaz, 2009; Séré, 2002); no obstante, aunque esta es una condición necesaria no se considera suficiente, ya que la comprensión de la naturaleza de la ciencia (NDC) no ocurre de manera espontánea, sino que amerita de un proceso explícito y reflexivo (Acevedo Díaz, 2008, 2009; Akerson, Abd-El-Khalik y Lederman, 2000; Lederman, 2006; Sandoval, 2003).

Por una parte, una forma de realizar un acercamiento del estudiante a la actividad científica investigativa es a través de la resolución de problemas en el laboratorio, ya que esto implica un proceso en el que se debe planificar, hipotetizar, recoger e interpretar datos, reflexionar e inferir, entre otros tipos de procesos pertinentes; por otra parte, la resolución de problemas en el laboratorio brinda un espacio para la reflexión epistemológica. Al respecto, Hodson (1994) plantea que el aprendizaje de la NDC se puede abordar considerando las siguientes cuatro fases básicas, inseparables e interactivas de la ciencia: (a) diseño y planificación, (b) realización práctica para la recolección de datos, (c) reflexión para evaluar los hallazgos y (d) registro y elaboración de informe para la comunicación respectiva. Esto le brinda al estudiante la oportunidad de involucrarse de manera dinámica, crítica y reflexivamente en el proceso de resolución de problemas en el laboratorio, en vez de seguir un protocolo recetario sin significado y sentido real que conduciría a fortalecer la idea ingenua sobre la existencia de un método científico único, estructurado algorítmicamente en pasos lineales y rígidos, como dogma metodológico de la investigación científica (Flores, Caballero y Moreira, 2013a; Flores-Espejo, 2014, 2018).

Al contrario de la visión recetaria de los trabajos prácticos en el laboratorio didáctico de ciencias, la resolución de problemas permite abordar situaciones experimentales a través de tres tipos de experimentos, de acuerdo con Etkina, Murthy y Zou (2006): (a) experimentos observacionales, usados cuando el fenómeno de estudio es desconocido por el 
estudiante, por lo que se coleccionan datos para encontrar patrones; (b) experimentos de corroboración, usados para contrastar hipótesis sobre la base de patrones en datos ya conocidos; y (c) experimentos de aplicación, usados para resolver un problema real sobre la base de la aplicación de conocimientos conceptuales que ya tiene el estudiante al respecto.

Este último tipo de trabajo experimental se ha venido implementando en el laboratorio de Bioquímica del Instituto Pedagógico de Caracas (IPC) desde inicio de la pasada década (Flores, Caballero y Moreira, 2009), mejorando progresivamente su implementación a través de enfoques didácticos constructivistas, particularmente el laboratorio con enfoque investigativo (similar al aprendizaje basado en proyecto) y el laboratorio con enfoque epistemológico usando el diagrama $\mathrm{V}$, según Moreira y Levandowski (1983). En esta avanzada didáctica y como resultado progresivo de la experiencia docente, esta cátedra ha logrado implementar un enfoque epistemológico-constructivista del laboratorio con el diagrama $\mathrm{V}$ para potenciar el aprendizaje significativo hacia la autonomía del estudiante a través de su empoderamiento en la integración de su pensar, hacer y sentir en situaciones problemáticas reales (Flores-Espejo, 2014).

El diagrama V se representa en la Figura 1 con sus elementos constitutivos básicos (Gowin y Álvarez, 2005):

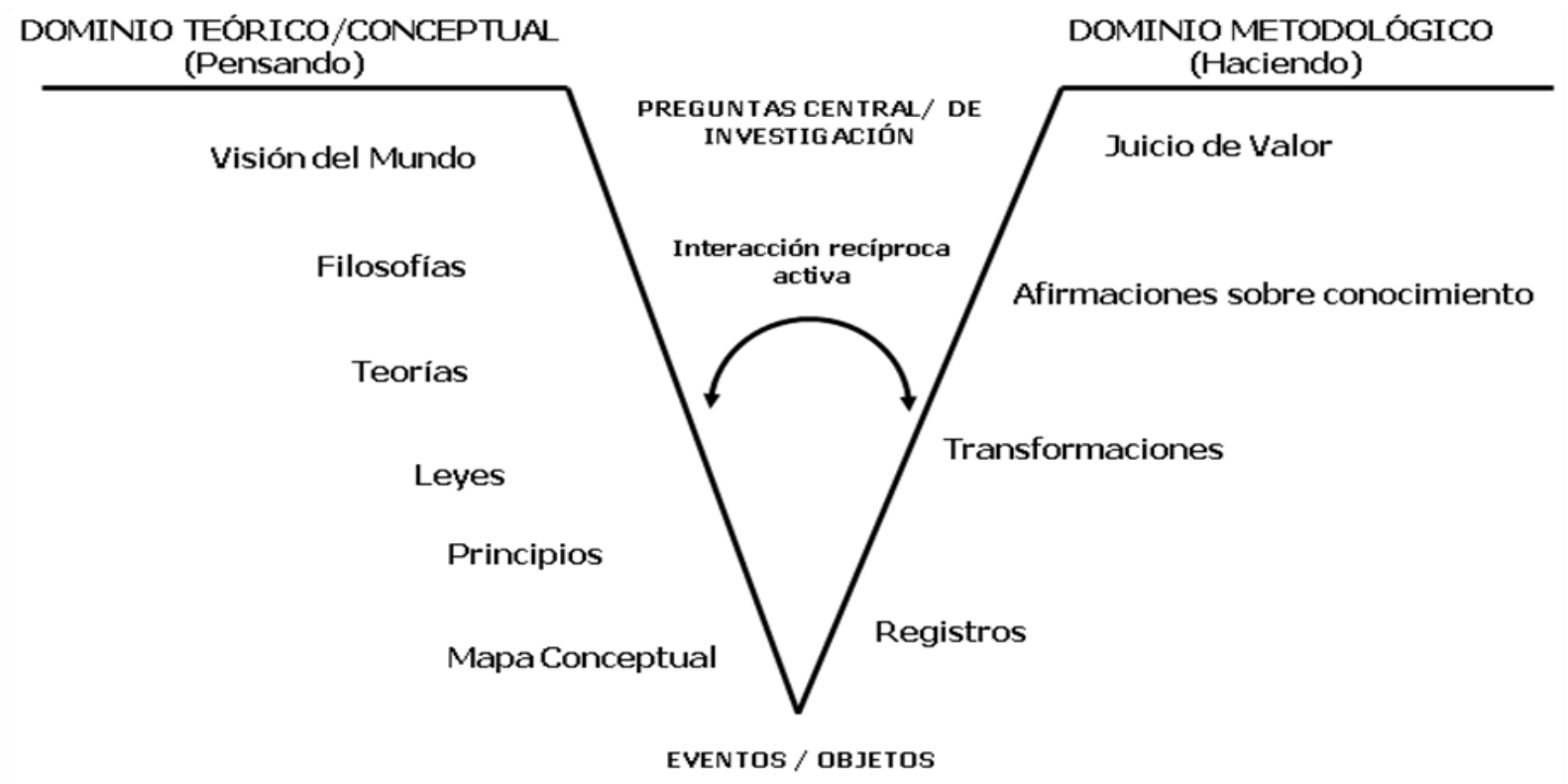

Figura 1. Elementos epistémicos básicos del diagrama $V$ que revelan la estructura del conocimiento. (Fuente: B. D. Gowin y M. C. Álvarez (2005). The art of educating with V diagrams. Cambridge: Cambridge University Press). 
En este sentido, el diagrama $\mathrm{V}$ de Gowin se usa como una herramienta heurística que permite la planificación y desarrollo de una investigación, considerando los aspectos teóricos y metodológicos de manera dinámica e interactiva en la búsqueda de respuestas a preguntas relacionadas con un evento de estudio (Flores, Caballero y Moreira, 2009; Flores, Caballero y Moreira, 2011; Gowin y Álvarez, 2005; Moreira, 2000, 2006; Moreira y Levandowski, 1983; Novak, 1979; Novak y Gowin, 1988).

Bajo el enfoque epistemológicoconstructivista se utiliza un proceso de reflexión epistemológica, la cual puede ser orientada a través de la sección denominada filosofía del diagrama $\mathrm{V}$ desde diferentes perspectivas. Según Gowin y Álvarez (2005), esta sección permite la expresión de las creencias sobre la naturaleza del conocimiento y del conocer que orienta o guía la indagación o proceso de investigación en la cual se está participando, lo que pudiera entenderse como un espacio para que el estudiante exprese sus ideas sobre la NDC en el contexto de los trabajos prácticos de laboratorio (TPL) que le permitan acercarse a la investigación científica real, al menos como ciencia aplicada, en el entendido paradigmático de una ciencia normal, en términos kuhnianos. Por lo tanto, el diagrama $\mathrm{V}$ brinda un espacio para tal reflexión de manera explícita, así como también constituye una vía para investigar al respecto.

En este contexto surgieron las siguientes preguntas que orientaron la presente investigación: (a) ¿cuáles aspectos de la investigación científica consideran los estudiantes importantes para reflexionar al respecto en el contexto de sus TPL?, (b) ¿qué tipo de enunciados (declaraciones, proposiciones) epistemológicas plantean los estudiantes en el contexto del desarrollo de sus TPL y (c) ¿cómo abordan los estudiantes un proceso de explicitación reflexiva de enunciados epistemológicos sobre la ciencia? Estas preguntas tienen sentido partiendo de la premisa de que los estudiantes reciben poca o ninguna atención académica obligatoria que esté orientada hacia un proceso de reflexión epistemológica sobre aspectos de la NDC en la mayoría de los cursos de ciencias de nuestra institución.

En este orden de ideas, la cátedra de Bioquímica de la UPEL-IPC, reconociendo la importancia de introducir a los estudiantes en un proceso de reflexión epistemológica, decidió realizar un proceso de acercamiento a este tipo de experiencia a través de la construcción libre de enunciados epistemológicos sobre la investigación científica de manera reflexiva y explícita en el contexto de los TPL desarrollados como problemas de investigación. En esta primera experiencia se centró la atención en los estudiantes de Química, como estudiantes avanzados, ya que cursan Bioquímica en el séptimo semestre de una carrera de diez semestres de acuerdo con el diseño curricular 1996.

Para ello, la cátedra de Bioquímica implementó el uso del diagrama $\mathrm{V}$ en el laboratorio para la resolución de problemas de interés planteados por los estudiantes dentro del contexto de la disciplina, recursos disponibles y tiempo de ejecución requerido; estos TPL se pueden ubicar como experimentos de aplicación principalmente, según la clasificación de Etkina et al. (2006). En este contexto se buscó responder al siguiente problema de investigación: ¿Cómo interpretar el proceso de construcción de enunciados epistemológicos sobre la ciencia que realizan los estudiantes en el contexto de desarrollo de TPL de Bioquímica centrados en la resolución de problemas de interés propio? 
Para responder a esta interrogante central se formuló el siguiente objetivo general: Caracterizar el proceso de construcción de enunciados epistemológicos sobre la ciencia que realizan los docentes en formación del área de Química en el contexto del desarrollo de los trabajos prácticos del laboratorio de Bioquímica como resolución de problemas de investigación con el diagrama $\mathrm{V}$.

Para responder al objetivo general se plantearon los siguientes objetivos específicos:

1. Categorizar los enunciados construidos en la sección de filosofía del diagrama $\mathrm{V}$ por los docentes en formación del área de Química participantes del laboratorio de Bioquímica.

2. Describir las características del proceso constructivo de los enunciados epistemológicos de los docentes en formación del área de Química participantes del laboratorio de Bioquímica.

3. Identificar los aspectos conceptuales de la ciencia considerados en la reflexión epistemológica de los docentes en formación del área de Química participantes del laboratorio de Bioquímica.

4. Identificar la posible tendencia epistemológica general del contenido de los enunciados epistemológicos elaborados por los docentes en formación del área de Química participantes del laboratorio de Bioquímica.

Esta investigación se justifica por tres razones principales: (a) no se encontraron estudios relacionados con las ideas manifestadas por los estudiantes sobre la ciencia como resultado de sus propias construcciones de enunciados epistemológicos en un contexto de investigación, (b) se desconocen las características del proceso constructivo de enunciados epistemológicos por parte de los estudiantes en un contexto de investigación y (c) la Cátedra de Bioquímica está comprometida con una formación docente que incluya el aprendizaje sobre ciencia, en términos hodsonianos, por lo que es necesario explorar diferentes alternativas didácticas al respecto para abrir un camino que permita avanzar hacia la búsqueda de soluciones sobre la problemática de la comprensión de la NDC en nuestro ámbito educativo.

\section{Referencial teórico}

Tomando en cuenta el planteamiento de Adúriz-Bravo (2007) en cuanto a que hay tres finalidades de la NDC: intrínseca, cultural e instrumental, parte de este trabajo es ubicable dentro de la primera, como proceso de reflexión, cuyo espacio se brinda al estudiante a través del uso del diagrama $\mathrm{V}$, sin pretensión de modificación alguna de las ideas deformadas, como una aproximación diagnóstica a dicha situación en el laboratorio de Bioquímica de la UPEL-IPC.

Es importante destacar que las concepciones sobre la ciencia, en general, se pueden estudiar a través de dos referenciales teóricos principales (Flores et al., 2013a): (a) naturaleza de la ciencia y (b) epistemología personal, de acuerdo con los aportes de Havdala y Ashkenazi (2007).

En el primer caso, la naturaleza de la ciencia se desarrolla bajo dos perspectivas (Acevedo Díaz, 2008) que en ambos caso abordan las concepciones relacionadas con la ciencia profesional, es decir, la epistemología científica (Sandoval, 2003): (a) la perspectiva de Lederman, que implica una posición más hacia la epistemología de la ciencia; y (b) la perspectiva del movimiento Ciencia, Tecnología y Sociedad (CTS), que tiene una posición más amplia, incluyendo los aspectos de la sociología de la ciencia. En ambos casos 
se busca conocer las ideas del estudiante sobre el conocimiento distal ("distal knowledge"), sobre la ciencia profesional.

En el segundo caso, la epistemología personal es un constructo complejo y confuso (Castañeda Figueiras y Peñalosa Castro, 2010) en vías de una definición clara (Elby, 2009), aborda las creencias sobre la naturaleza del conocimiento y del conocer del propio individuo; incluye las creencias sobre el aprendizaje y la enseñanza, a pesar de la polémica definitoria que ha habido al respecto. Se ha abordado en la literatura bajo diferentes nombres, tales como creencias epistemológicas, juicio reflexivo, reflexión epistemológica, modos de conocer, teorías epistemológicas, creencias epistémicas $\mathbf{0}$ recursos epistemológicos. La epistemología personal se relaciona con el denominado conocimiento proximal ("proximal knowledge"), que implica la comprensión que el individuo tiene de su propio aprendizaje de la ciencia (Hogan, 2000).

Cabe señalar que este trabajo se enmarca dentro de la teoría del aprendizaje significativo de David Ausubel, en virtud de que busca explorar el sustrato conceptual de los enunciados epistemológicos que construyen los estudiantes en el contexto de los TPL que desarrollan. En este sentido, se destaca el trabajo de Flores, Caballero y Moreira (2013b), el cual permite una interpretación de esta teoría en el contexto del laboratorio de ciencias.

Ahora bien, en virtud de que las ideas epistemológicas que pueden reflejar los estudiantes constituyen parte de su cosmovisión, se toma como referencia a Cobern (2000). Al respecto, este autor plantea que la visión de mundo "está compuesta de esas ideas fundamentales que uno simplemente toma por garantizadas (...) porque son aprendidas y sostenidas culturalmente, y se ha encontrado a través de la experiencia diaria que son viables" (p. 237); señala, además, que estas creencias o supuestas ideas son elementos cognitivos que subyacen a las construcciones epistemológicas, así como las conceptuales y que la propia ciencia se apoya en bases presuposicionales en el sentido de que asume que el mundo natural es accesible a la mente humana desde el punto de vista ontológico, epistemológico y axiológico, de modo que todo conocimiento se fundamenta en última instancia en supuestos que implican en sí una creencia; asimismo indica que la distinción epistémica entre conocimiento y creencia puede tornarse ambigua, ya que un conocimiento es una creencia razonada. Esto revela la complejidad de esta temática que parece ineludible para el docente de ciencias que desea avanzar hacia niveles de compromiso formativo de sus estudiantes, tomando en cuenta que no es suficiente con que aprendan ciencia y aprendan a hacer ciencia, sino que es necesario aprender sobre ciencia, es decir, sobre su naturaleza, como lo señala Hodson (1994).

\section{MÉTODO}

Esta investigación se considera un estudio de caso exploratorio de tipo cualitativo. Se llevó a cabo con 8 estudiantes de profesorado de Química (6 de sexo femenino y 2 de sexo masculino), quienes formaron parte de un grupo de laboratorio de Bioquímica Los participantes fueron organizados por pares en cuatro equipos para la realización de los TPL y fueron identificados como: $1 \mathrm{AB}, 2 \mathrm{AB}, 3 \mathrm{AB}$ y $4 \mathrm{AB}$ ).

El diseño de la investigación asumido se resume en la Tabla 1 : 
Tabla 1. Diseño de la investigación

\begin{tabular}{|c|c|c|c|c|c|}
\hline Muestra & $\begin{array}{l}\text { Momento } \\
\text { de } \\
\text { desarrollo }\end{array}$ & Método & $\begin{array}{l}\text { Instrumentos } \\
\text { empleados }\end{array}$ & Fuente de datos & $\begin{array}{c}\text { Procesamiento de } \\
\text { datos }\end{array}$ \\
\hline $\begin{array}{l}\text { Ocho (8) } \\
\text { docentes en } \\
\text { formación } \\
\text { del área de } \\
\text { Química, } \\
\text { cursantes del } \\
\text { laboratorio } \\
\text { de } \\
\text { Bioquímica } \\
\text { en la UPEL- } \\
\text { IPC }\end{array}$ & $\begin{array}{l}\text { Durante el } \\
\text { desarrollo } \\
\text { de los } \\
\text { trabajos } \\
\text { prácticos de } \\
\text { laboratorio }\end{array}$ & $\begin{array}{l}\text { Observación } \\
\text { participante en } \\
\text { construcción de } \\
\text { enunciados } \\
\text { epistemológicos } \\
\text { por los } \\
\text { estudiantes }\end{array}$ & $\begin{array}{l}\text { Diagrama V } \\
\text { Registro } \\
\text { anecdótico }\end{array}$ & $\begin{array}{l}\text { Enunciados } \\
\text { epistemológicos } \\
\text { escritos sobre la } \\
\text { ciencia en la } \\
\text { sección de } \\
\text { filosofía del } \\
\text { diagrama V }\end{array}$ & $\begin{array}{l}\text { Análisis de } \\
\text { contenido en } \\
\text { búsqueda de: (a) } \\
\text { categorías } \\
\text { emergentes, (b) } \\
\text { conceptos } \\
\text { utilizados y (c) } \\
\text { tendencias } \\
\text { epistemológicas. } \\
\text { Análisis } \\
\text { descriptivo } \\
\text { complementario } \\
\text { por frecuencias }\end{array}$ \\
\hline
\end{tabular}

Esta investigación se enmarcó de modo general dentro de la línea teórica de la NDC, considerando tres aspectos: (a) la búsqueda de alternativas metodológicas en la evaluación de las ideas epistemológicas de los estudiantes, (b) el análisis de enunciados epistemológicos y (c) la experiencia docente sobre el proceso de construcción reflexiva de aspectos epistemológicos de la ciencia que realizan los estudiantes en el contexto de los TPL que desarrollan.

Esta investigación se encuentra dentro del paradigma hermenéutico bajo un enfoque fenomenológico con el empleo de un análisis de contenido para interpretar y comprender la realidad problematizada, aplicando un análisis por ideas, en correspondencia con lo planteado por Barrera (2007). Se realiza una complementariedad descriptiva para fines de una mejor comprensión del fenómeno de estudio en la que hubo observación participante.

\section{Contexto didáctico de la investigación}

El término "enunciado" en esta investigación se refiere a una expresión, planteamiento o proposición, que puede ser adecuada o inadecuada en relación con el contenido que expresa; en este caso, un enunciado epistemológico sobre la ciencia se asumirá como una idea elaborada por el estudiante acerca de algún aspecto de la ciencia, tomando como contexto el TPL que desarrolla como resolución de un problema de investigación, por lo que implica una visión, idea, creencia o concepción, generalmente inmersa en una cosmovisión.

El objetivo didáctico general desarrollado en el laboratorio fue el siguiente: favorecer en los estudiantes la construcción consciente, explícita y reflexiva de enunciados epistemológicas sobre la ciencia, considerando la investigación científica como eje reflexivo, en el contexto de los TPL planteados como resolución de problemas de investigación con el uso del diagrama $V$.

Se desarrollaron cinco (5) TPL de complejidad creciente con contenidos disciplinares propios del curso de Bioquímica, aplicados a situaciones problemáticas planteadas por los estudiantes sobre la base de su interés. Se usó un diagrama $\mathrm{V}$ con 
información orientadora que describía cada una de las partes de este heurístico (diagrama $\mathrm{V}$ orientador) y que fue validado antes de su aplicación por más de seis (6) semestres consecutivos en el laboratorio de Bioquímica del IPC; fue actualizado en su sección de filosofía y visión del mundo, según criterios de Gowin y Álvarez (2005), para su implementación en el período académico en que se realizó el presente estudio.

Los temas de los 5 TPL desarrollados fueron los siguientes: (a) identificación de macromoléculas; $\quad$ (b) cuantificación espectrofotométrica de una proteína; (c) extracción, caracterización y cuantificación de un carbohidrato; (d) extracción, identificación y cuantificación de lípidos; y (e) estudio catalítico de una enzima. Se consideró un ambiente didáctico de actividades abiertas de laboratorio bajo una orientación docente crítica para la integración de aspectos teóricos y metodológicos hacia la solución de los problemas de investigación planteados.

Cada TPL contempló 4 fases (Flores et al., 2009): (a) fase preparatoria: búsqueda de información previa necesaria para formular el problema de investigación; (b) fase preexperimental: discusión y precisión en consenso del problema a resolver; elaboración de la primera versión del marco teórico/conceptual y el metodológico con procedimiento $\mathrm{y}$ tablas de registros prediseñadas en el diagrama V; (c) fase experimental: desarrollo del TPL con apoyo en versión de avance del diagrama $\mathrm{V}$ y ajuste metodológico en caso necesario); y (d) fase post-experimental: elaboración de versión final del diagrama $\mathrm{V}$ como informe de cierre. Cada TPL requirió dos semanas, excepto el último que dispuso de 4 semanas por su nivel de complejidad y grado de independencia.

\section{Procedimiento}

La sección de filosofía del diagrama $\mathrm{V}$ se orientó hacia la construcción libre de enunciados epistemológicos sobre la ciencia, tomando como eje reflexivo la investigación científica, sobre la base de aspectos que tuvieran sentido para cada estudiante en el contexto de los TPL desarrollados. Los enunciados se comenzaron a elaborar desde la fase preparatoria del laboratorio hasta la fase post-experimental con entrega del informe final en diagrama $\mathrm{V}$, permitiendo así la reflexión y mejoramiento de las ideas expresadas durante el desarrollo de cada TPL. La investigadora realizó registros de observación de lo que se consideró relevante en este proceso exploratorio.

La investigación se desarrolló a través del siguiente procedimiento general: (a) realización de un taller sobre el uso del diagrama $\mathrm{V}$ en el laboratorio; (b) desarrollo de los TPL usando el diagrama $\mathrm{V}$ para resolver problemas en el contexto disciplinar; (c) orientación general al grupo sobre la construcción de enunciados epistemológicos sobre la ciencia; (d) orientación particular a cada equipo de estudiantes sobre el proceso constructivo de enunciados epistemológicos de acuerdo con la necesidad planteada; (e) transcripción de los datos de la sección de filosofía del diagrama V; (f) análisis de contenido de los enunciados elaborados, (g) categorización de los enunciados y (h) validación de las categorías a través de juicio de tres expertos.

\section{Análisis de datos}

La fuente de datos provino de la sección de filosofía del diagrama $\mathrm{V}$ como vertiente declarativa con contexto disciplinar, en la que los estudiantes escribieron los enunciados 
epistemológicos sobre la ciencia que construyeron durante el desarrollo de los TPL. Cada enunciado fue analizado para determinar si respondía, según lo esperado, a la categoría de epistemológico; en caso, contrario se le ubicó en categorías emergentes que se consideraron pertinentes a su contenido y se discriminaron de acuerdo con su nivel de abstracción y diferenciación conceptual en función de los criterios de muy bajo, bajo, medio, alto y muy alto. La categorización de los enunciados elaborados fue validada a través del juicio de tres docentes - investigadores del área de ciencias; la posible tendencia epistemológica se realizó en función de contemporánea o tradicional, según Flores et al. (2013a)

\section{RESULTADOS}

Los resultados se presentaron de acuerdo con los objetivos específicos planteados en esta investigación: (a) categorización de los enunciados elaborados, (b) características del proceso constructivo de los enunciados epistemológicos, (c) aspectos conceptuales de la ciencia considerados en la reflexión y (d) tendencias epistemológicas generales apreciables del contenido de los enunciados epistemológicos.

\section{Categorización de los enunciados elaborados}

El proceso de categorización condujo a la discriminación de 4 categorías de enunciados construidos por los estudiantes de la siguiente manera:

- Enunciado sobre la visión del mundo (VM)

- Enunciados epistemológicos sobre la ciencia (EE)

- Enunciados de conocimiento científico (EC)

- Enunciados sobre el aprendizaje (EA)

Estas categorías se discriminaron según la descripción dada en la Tabla 2; los VM y EE se describieron en el diagrama $\mathrm{V}$ orientador antes de iniciarse el proceso de explicitación de enunciados, pero los EC y EA emergieron durante el proceso, procediéndose a su descripción durante el proceso constructivo. Se aclara que los enunciados expresados por los estudiantes como EA pudieron interpretarse como creencias sobre el aprendizaje, a la luz del constructo de epistemología personal; sin embargo, esto no constituyó el propósito de este trabajo, ya que el interés estaba centrado en los enunciados epistemológicos. 
Tabla 2. Descripción de los criterios usados para categorizar los enunciados elaborados en la sección de filosofía del diagrama $\mathrm{V}$ por los estudiantes.

\section{ENUNCIADOS ELABORADOS}

VISIÓN DEL MUNDO

$(\mathrm{VM})$

ENUNCIADOS EPISTEMOLÓGICOS

\section{ENUNCIADOS DE CONOCIMIENTO}

CIENTÍFICO

(EC)

ENUNCIADO SOBRE EL APRENDIZAJE

(EA)

Sobre la base de los criterios considerados en la Tabla 2, se clasificaron los enunciados de los estudiantes, los cuales se muestran en la Tabla 3.
Simplifica la complejidad y se refiere a creencias que no tienen que debatirse; son ciertas para el individuo y lo hacen sentir seguro de ellas por creer en lo que afirma. Refleja una manera de ver el mundo (Gowin y Álvarez, 2005 , pp. 36, 51) considerando el contexto de estudio; las creencias subyacen lo que se pretende investigar. Motivan y guían la investigación. Ejemplo: (a) "la naturaleza es ordenada y cognoscible" (Novak y Gowin, 1988, p.77).

La filosofía no toma nada por garantizado: hace lo simple complejo y lo complejo simple (Gowin y Álvarez, 2005, p. 36). Se expresa como una declaración que puede ser argumentable, a diferencia de lo que ocurre con la VM, concerniente a algún aspecto de la investigación científica que se considere pertinente en el contexto del TPL que se desarrolla. Ejemplo: “El conocimiento es más que la recolección de datos” (Gowin y Álvarez, 2005, p. 82).

Se refieren a contenidos teóricos/conceptuales disciplinares pertinentes al trabajo práctico de laboratorio que se desarrolla. Son expresiones relacionadas con el tema de estudio del problema. Ejemplo: "Las proteínas son macromoléculas esenciales para la vida".

Son enunciados derivados de teorías psicológicas del aprendizaje o pueden ser creencias formuladas por los estudiantes sobre el aprendizaje. Un ejemplo: "los estudiantes aprenden activamente participando" (Gowin y Álvarez, 2005, p. 80); aunque esta expresión es ejemplificada por los autores en su libro, en la sección de filosofía de un diagrama $\mathrm{V}$, en el contexto de este trabajo de investigación se consideró un EA. 
Tabla 3. Planteamientos elaborados en cada trabajo práctico de laboratorio

\begin{tabular}{|c|c|c|c|c|c|}
\hline TPL & $\begin{array}{l}\text { 1. IDENTIFICACIÓN DE } \\
\text { MACROMOLÉCULAS EN } \\
\text { DISOLUCIÓN }\end{array}$ & $\begin{array}{l}\text { 2. CUANTIFICACIÓN } \\
\text { ESPECTROFOTOMÉTRICA } \\
\text { DE PROTEÍNAS }\end{array}$ & $\begin{array}{l}\text { 3. EXTRACCIÓN, } \\
\text { CARACTERIZACIÓN Y } \\
\text { CUANTIFICACIÓN DE } \\
\text { CARBOHIDRATOS }\end{array}$ & $\begin{array}{l}\text { 4. EXTRACCIÓN, } \\
\text { IDENTIFICACIÓN Y } \\
\text { CUANTIFICACIÓN DE UN } \\
\text { LÍPIDO }\end{array}$ & $\begin{array}{l}\text { 5. ESTUDIO CATALÍTICO } \\
\text { DE UNA ENZIMA }\end{array}$ \\
\hline $1 \mathrm{AB}$ & $\begin{array}{l}\text { El aprendizaje es producto de } \\
\text { una educación y una formación } \\
\text { en donde los individuos pueden } \\
\text { especular y sustentar creencias } \\
\text { que van más allá del } \\
\text { conocimiento. [EA] }\end{array}$ & $\begin{array}{l}\text { El estudio de las biomoléculas } \\
\text { permite conocernos a nosotros } \\
\text { mismos, es decir, conocer las } \\
\text { biomoléculas es conocer la } \\
\text { esencia de la vida. [EC] }\end{array}$ & $\begin{array}{l}\text { El estudio de la trehalosa } \\
\text { permite convertir el } \\
\text { aprendizaje en ventajas } \\
\text { competitivas en beneficio } \\
\text { de la humanidad. [EA] }\end{array}$ & $\begin{array}{l}\text { El estudio de la yema de } \\
\text { huevo de gallina emprende } \\
\text { la curiosidad en los que tal } \\
\text { vez no son científicos [VM], } \\
\text { incitando así a la lectura y } \\
\text { leer es aprender de las } \\
\text { páginas escritas. [EA] }\end{array}$ & $\begin{array}{l}\text { Solucionar dificultades } \\
\text { experimentales anima a } \\
\text { inventar el mañana sin } \\
\text { olvidar el justo valor del } \\
\text { ayer. [EE] }\end{array}$ \\
\hline $2 \mathrm{AB}$ & $\begin{array}{l}\text { La bioquímica es un conjunto de } \\
\text { acciones encaminadas y } \\
\text { dirigidas hacia determinado fin, } \\
\text { el cual es el de obtener un } \\
\text { conocimiento verificable sobre } \\
\text { los hechos que le ocurre al ser } \\
\text { humano, para poder dar } \\
\text { explicación a la lógica molecular } \\
\text { de la vida [EE], donde las } \\
\text { macromoléculas forman parte } \\
\text { de la creación del ser humano. } \\
{[\mathrm{VM}]}\end{array}$ & $\begin{array}{l}\text { La experimentación es esencial } \\
\text { para el conocimiento profundo } \\
\text { de la base genética de los seres } \\
\text { vivos. [EE] }\end{array}$ & $\begin{array}{l}\text { No se crea nada en un } \\
\text { sentido absoluto, sólo se } \\
\text { descubren las maravillas } \\
\text { del mundo, buscando } \\
\text { soluciones y explicaciones } \\
\text { a los problemas que } \\
\text { aparecen a lo largo de la } \\
\text { historia humana. [EE] }\end{array}$ & $\begin{array}{l}\text { La esencia del } \\
\text { experimentador está en ver } \\
\text { más allá de lo fisicamente } \\
\text { palpable. [EE] Las } \\
\text { experiencias que se van } \\
\text { adquiriendo en el recorrido } \\
\text { de nuestra vida permiten } \\
\text { enriquecer ros } \\
\text { conocimientos de los cuales } \\
\text { hacemos uso en la resolución } \\
\text { de posibles problemas que se } \\
\text { puedan presentar. [EE] }\end{array}$ & $\begin{array}{l}\text { El científico no crea nada en } \\
\text { sentido absoluto, sólo } \\
\text { descubre las maravillas del } \\
\text { mundo y desarrolla } \\
\text { soluciones y explicaciones a } \\
\text { los problemas que van } \\
\text { apareciendo a lo largo de la } \\
\text { historia humana [EE]. El } \\
\text { hombre tiene un reto } \\
\text { permanente de encontrar } \\
\text { nuevas respuestas a sus } \\
\text { necesidades [VM], } \\
\text { aplicando hoy algo que } \\
\text { aprendió ayer, basándose } \\
\text { en lo que otro había hecho } \\
\text { anteayer [EA]. }\end{array}$ \\
\hline
\end{tabular}




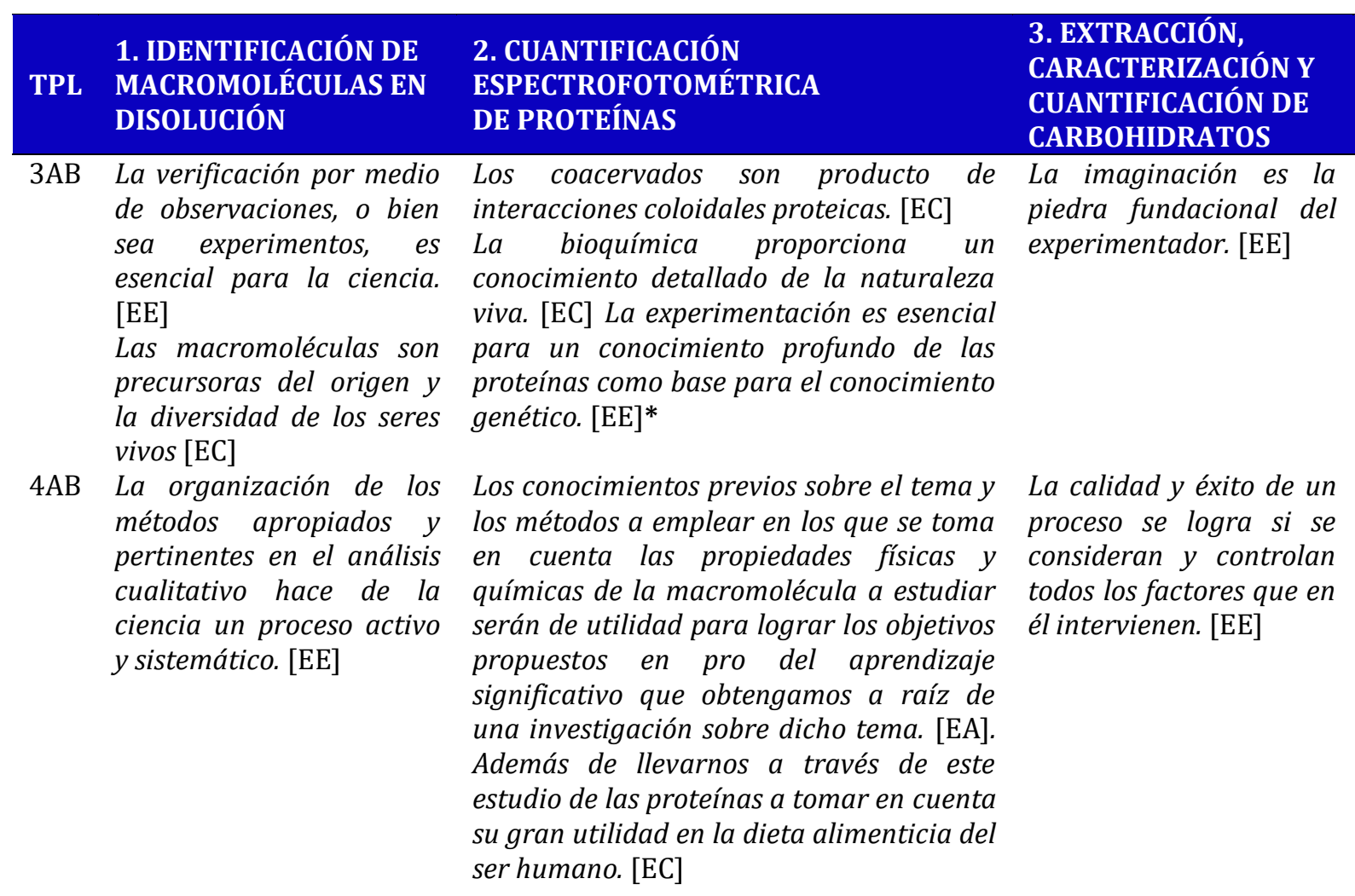

3AB La verificación por medio Los coacervados son producto de de observaciones, o bien interacciones coloidales proteicas. [EC]

sea experimentos, es La bioquímica proporciona un Las macromoléculas son vivos [EC] pertinentes en el análisis cualitativo hace de la ciencia un proceso activo y sistemático. $[\mathrm{EE}]$

4. EXTRACCIÓN,

IDENTIFICACIÓN

CUANTIFICACIÓN DE

UN LÍPIDO

experimentación. [EE]

¿Quién dijo que la

ciencia tiene la

respuesta de todo? [EE]
El conocimiento rompe

las barreras de la mera

Por medio del El estudio de la acción pensamiento analítico- enzimática (en este caso de la crítico se puede catalasa) permite establecer incrementar el valor de conocimientos en el área de la la experiencia para medicina [EC],con la finalidad resolver problemas de de mejorar la calidad de vida modo satisfactorio. [EE] de las personas [EE]. Los experimentos de laboratorio, así como las observaciones, análisis y determinaciones realizadas sobre la acción enzimática permiten confirmar y verificar los conocimientos que se tienen sobre el tema. [EE]

VM: visión del mundo; EC: planteamientos de conocimiento científica; EA: enunciado sobre el aprendizaje; EE: enunciados epistemológicos sobre la investigación científica.

Nota. Los EE marcados con "asteriscos" $\left(^{*}\right)$ reflejan el contexto disciplinar específico del TPL 
En la Tabla 4 se presentan las respectivas categorizaciones realizadas de los enunciados construidos por cada equipo de estudiantes.

Tabla 4. Categorización de los enunciados construidos por los estudiantes en cada TPL

\begin{tabular}{|c|c|c|c|c|}
\hline \multirow{2}{*}{ TPL } & \multicolumn{4}{|c|}{ TIPOS DE ENUNCIADOS ELABORADOS POR PARES DE ESTUDIANTES } \\
\hline & $1 \mathrm{AB}$ & $2 \mathrm{AB}$ & $3 \mathrm{AB}$ & $4 \mathrm{AB}$ \\
\hline 1 & EA & EE, VM & EE; EC & EE \\
\hline 2 & EC & EE & EC; EC; $\mathbf{E E}$ & PA; EC \\
\hline 3 & EA & EE & EE & $\mathbf{E E}$ \\
\hline 4 & VM, EA & EE; EE & EE; EE & EE \\
\hline 5 & $\mathbf{E E}$ & EE; VM, EA & VM & EC, EE; EE \\
\hline
\end{tabular}

VM, visión de mundo; EC, enunciado de conocimiento científico; EA, enunciado sobre aprendizaje; EE, enunciado epistemológico sobre la ciencia. La "coma" indica la presencia de enunciaos diferentes identificables en la misma construcción gramatical; el "punto y coma" indica enunciados en construcciones gramaticales por separado.

\section{Características del proceso constructivo de enunciados epistemológicos}

Este proceso resultó muy dinámico debido a que fueron surgiendo ideas de diferente naturaleza que no respondían a lo solicitado específicamente, siendo necesario esclarecerlas y discriminarlas, lo cual fue una tarea de reflexión permanente en la que se observaron respuestas diferenciales de parte de los equipos de estudiantes. El proceso reveló que la elaboración de enunciados epistemológicos implica discriminarlos conceptualmente de manera consciente de: (a) las creencias que conforman la visión del mundo, proceso que requiere de imaginación como técnica de trabajo, como lo señalan Gowin y Álvarez (2005); y (b) del conocimiento científico, considerando que las afirmaciones científicas se anidan en contextos filosóficos.

La dificultad de los estudiantes para discriminar filosofía de creencia y de ciencia es una situación esperada en este contexto, especialmente si se parte del hecho de que esto no constituye una práctica reflexiva durante la formación docente, como todos ellos lo manifestaron durante las discusiones al respecto, por lo que la dificultad comienza a aflorar cuando las creencias se empiezan a explicitar y a discriminar de otras ideas en construcción como los enunciados filosóficos y el conocimiento científico que debe precisar el estudiante en cada trabajo de laboratorio. Particularmente, la distinción epistémica entre conocimiento y creencia puede tornarse ambigua en ciertos enunciados, ya que como este autor señala "el conocimiento sin fe carece de fundamento; la creencia sin conocimiento es vacía" (p. 223).

Para facilitar este intento constructivo se hizo necesario discriminar con la orientación docente las ideas frecuentemente confundidas durante el proceso, referidas a VM, EC y EA. Al darse cuenta los estudiantes de que no habían construido un EE, sino otro tipo de enunciado, reorientaban sus esfuerzos para lograr la construcción solicitada, lo que requería de un esfuerzo reflexivo mayor.

En virtud de las dificultades inicialmente observadas y para contribuir a superar esta situación, la docente-investigadora realizó discusiones en grupo y por pares de estudiantes y sugirió incorporar al diagrama $\mathrm{V}$ una sección denominada Introducción en el segundo TPL a fin de colocar allí los planteamientos del tipo EC que tendían a 
confundirse principalmente con EE; esta sección se ubicó al lado izquierdo superior del diagrama $\mathrm{V}$, antes de la sección denominada visión del mundo, contribuyendo así a mejorar el proceso discriminatorio entre conocimiento científico y filosofía, así como entre conocimiento científico y creencias. En los casos en que los estudiantes planteaban una creencia en la sección de filosofía, se les sugería ubicarlo en la sección de visión del mundo del diagrama $\mathrm{V}$.

La sección de Introducción se refería a una breve reseña de aspectos científicos relevantes que permitiera brindar un soporte teórico referencial del estudio de manera global. Aunque esto sirvió de ayuda, se pudo apreciar que la dificultad de los estudiantes con relación a estas tres construcciones antes mencionadas se presentó de acuerdo con el siguiente orden en general: conocimiento científico < creencias $<$ enunciados epistemológicos, siendo en todo caso el aspecto filosófico el que resultaba más difícil de aprehender, por su alto nivel de abstracción, demandando así mayor tiempo y esfuerzo cognitivo. Esto es consistente con lo que Gowin y Álvarez (2005, p. 42) plantean sobre la filosofía que "esta actividad es un esfuerzo persistente para pensar bien cosas a través de asunciones, presuposiciones, o para clarificar argumento racional".

En relación con las creencias manifestadas es importante considerar lo señalado por Cobern (2000) por lo que esta visión se basa en presunciones bajo las cuales las personas actúan diariamente sin prestarle mucha atención hasta que son confrontadas con ellas. Sobre esta base, se puede entender, entonces, que estas ideas están implícitas en el pensamiento del individuo $y$ que son importantes de explicitar en este proceso constructivo de planteamientos epistemológicos bajo la orientación docente, como lo destaca Hodson (1994) en la siguiente expresión: "para asegurar que los estudiantes tengan éxito en el aprendizaje sobre la naturaleza de la ciencia, el primer paso necesario es convertir lo implícito en explícito" (p. 307); en esta experiencia se partió de una explicitación por parte del estudiante.

Un aspecto importante de mencionar es que los estudiantes del equipo $1 \mathrm{AB}$ (Tabla $3 \mathrm{y}$ 4) fueron los que más EA construyeron, revelando que tenían dificultades para construir EE enunciados, ya que confundieron inicialmente enunciados epistemológicos con enunciados psicológicos derivados de las teorías del aprendizaje. A pesar de que Gowin (2005) presenta enunciados sobre aprendizaje como ejemplos, en la sección de filosofía en su reciente obra, la orientación docente-investigadora en este trabajo trató de diferenciar entre aspectos psicológicas del aprendizaje y aspectos filosóficos del conocimiento científico, con el fin de que los estudiantes concentraran sus esfuerzos en reflexionar sobre aspectos epistemológicos propiamente dicho acerca de la naturaleza de la ciencia.

En la Tabla 5 se resumen las observaciones sobre el proceso constructivo de los enunciados epistemológicos de los estudiantes en cada trabajo práctico en esta experiencia exploratoria. 
Tabla 5. Descripción del proceso de construcción reflexiva de enunciados epistemológicos acerca de la ciencia en el contexto de los TPL desarrollados

$\begin{array}{ccc}\text { TÍTULO DE LOS TPL } & \text { DESCRIPCIÓN GENERAL DEL PROCESO } & \text { CARACTERÍSTICAS } \\ & \text { CONSTRUCTIVO } & \text { OBSERVABES DEL } \\ \end{array}$

1. Identificación de una macromolécula en disolución

2. Cuantificación espectrofotométri ca de una proteína

3. Extracción, caracterización y cuantificación de trehalosa

4. Extracción e identificación de lecitina y colesterol; cuantificación de colesterol

5. Estudio enzimática sobre la catalasa
La mayoría de los estudiantes confundían inicialmente creencias, filosofías y conocimiento científico, logrando construir uno solo.

El docente incorporó una sección adicional en el diagrama $\mathrm{V}$ que denominó introducción para posibilitar la diferenciación entre creencias y filosofía en relación con el conocimiento científico. Esto no permitió, de inmediato, mejorar la discriminación conceptual en la mayoría de los estudiantes. Algunos se pronunciaron en función del aprendizaje en vez de la naturaleza del conocimiento científico en sí.

Se observó un mejoramiento en la mayoría de los estudiantes en cuanto a la elaboración de enunciados epistemológicos.

Se apreció el avance relativo de la mayoría de los estudiantes en la elaboración de enunciados epistemológicos, excepto para el equipo $1 \mathrm{AB}$
Indiferenciación conceptual entre creencia, filosofía y conocimiento científico

Indiferenciación conceptual entre filosofía, conocimiento científico y enunciados psicológicos de aprendizaje.

Diferenciación conceptual progresiva de enunciados epistemológicos

Diferenciación conceptual progresiva de los enunciados epistemológicos.
Se continuó observando la elaboración de enunciados epistemológicos pero algunos resultaron todavía mezclados con creencias y conocimiento científico
Un aspecto que vale la pena resaltar aquí es el hecho de que los estudiantes se sentían muy satisfechos cuando lograban aproximarse a la explicitación de sus ideas sobre algún aspecto de la ciencia, las cuales usaban para establecer diálogos, conexiones jocosas y hacer cuestionamientos más profundos acerca de la ciencia en el contexto del TPL que desarrollaban. Un ejemplo de esto fue la expresión: "La imaginación es la piedra fundacional del experimentador" (ver Tabla 3, 3AB, TL \# 3), que refleja un contenido no empirista (en el sentido positivista del término) de la investigación científica. En la Tabla 6 se muestra el avance de cada par de estudiantes.

Tabla 6. Observaciones del proceso de construcción reflexiva de enunciados epistemológicos 
por cada equipo de estudiantes en el laboratorio

PARES DE

ESTUDIANTES
DESCRIPCIÓN GENERAL DEL PROCESO CONSTRUCTIVO POR EQUIPO DE ESTUDIANTES

Este equipo intentó elaborar planteamientos

$1 \mathrm{AB}$ epistemológicos, pero su logro fue muy bajo, ya que en todos los TPL plantearon creencias o conocimiento científico, logrando construir uno en el último TPL.

Este equipo hizo avances notorios en la elaboración de $2 \mathrm{AB}$ $3 \mathrm{AB}$ planteamientos epistemológicos, la cual se enmarcó progresivamente en un cierto nivel de abstracción a partir del segundo trabajo práctico.

Este equipo hizo avances apreciables llegando a elaborar planteamientos epistemológicos con un nivel de abstracción considerable en relación con el resto del grupo.

Este equipo hizo poco avances en la elaboración de planteamientos epistemológicos, aunque con poco nivel de abstracción y algo confusos.
NIVEL DE ABSTRACCIÓN RELATIVA DEL PROCESO

Muy bajo nivel de abstracción apreciable y poca diferenciación conceptual

Nivel medio de elaboración abstracta y de diferenciación conceptual

Alto nivel de elaboración abstracta y discriminación conceptual

Bajo nivel de elaboración abstracta y discriminación conceptual
Este proceso discriminativo tampoco fue sencillo para los estudiantes, posiblemente por el hecho de que hay aspectos que se entrecruzan entre filosofía de la ciencia y psicología del conocimiento que hace a veces difícil su comprensión. Al respecto, Japiassu (1991, pp. 33-35) hace mención de una epistemología psicológica que implica psicología del conocimiento científico, un tema que se torna complejo en su esencia misma. En todo caso, lo que se pretendía hacer era que los estudiantes estuvieran conscientes en cuanto a la diferencia entre la lógica del conocimiento y la psicología del conocimiento, es decir, entre, filosofía de la ciencia y aprendizaje de la ciencia. En este sentido, el diagrama $\mathrm{V}$ permitió la oportunidad de discutir sobre ambos aspectos y los estudiantes mejoraron en general en esta discriminación, volviéndose más críticos al respecto.

Tomando en cuenta lo antes planteado se puede apreciar en la Tabla 4 los EE formulados y los que responden a otros tipos de planteamientos, indicando esto que la confusión en el proceso de explicitación fue mayor para unos equipos de estudiantes que para otros, lo que es entendible debido a que estaban tratando de responder al proceso desde su propia comprensión, algo totalmente nuevo para ellos. Se pudiera decir que el número total de EE elaborados por cada equipo de estudiantes sería un criterio para ubicar el avance de cada uno, que posiblemente sería el siguiente: $\mathbf{2 A B}(6 \mathrm{EE})>$ $\mathbf{3 A B}(5 \mathrm{EE})=4 \mathbf{A B}(5 \mathrm{EE})>\mathbf{1 A B}(1 \mathrm{EE}) . \mathrm{Sin}$ embargo, para superar esta apariencia $\mathrm{y}$ sincerar el progreso constructivo real de los estudiantes se usaron dos criterios orientadores en la construcción de los EE, los cuales sirvieron de base para su análisis en esta investigación:

a. La discriminación conceptual de un EE, que se puede apreciar cuando el estudiante no mezcla su contenido con otros tipos de planteamientos como un VM, un EA o un EC. 
Un ejemplo de esto se muestra en la Tabla 4, TPL \# 1: el equipo 4AB presenta un EE sin otro tipo de planteamiento, mientras que el $2 \mathrm{AB}$ lo presenta mezclado con un $\mathrm{VM}$, el $3 \mathrm{AB}$ lo presenta seguido de un EC, mientras que el equipo $1 \mathrm{AB}$ construye un EA en vez de un EE.

b. El nivel de abstracción y complejidad, que implica el nivel de elaboración cognitiva para exponer ideas no fácilmente apreciables en el plano concreto de la realidad. Un ejemplo de esto se observa en el TPL \# 3 (ver Tabla 3): el equipo $3 \mathrm{AB}$ presenta un EE mucho más elaborado en abstracción y complejidad que el resto de los equipos. Se observó el siguiente orden creciente de complejidad y abstracción entre los planteamientos elaborados: EC $<$ EA $<$ VM $<$ EE, es decir, los estudiantes discriminaban más fácilmente un EC de un EE que un EA de un EE, siendo más difícil discriminar entre un VM y un EE, probablemente por su sentido más filosófico.

De este modo, se puede establecer el siguiente orden de logro constructivo de los enunciados epistemológicos por los estudiantes, tomando en cuenta la no mixtura con otros tipos de enunciados: $\mathbf{3 A B}$ (5 $\mathrm{EE})>2 \mathrm{AB} \quad(5 \mathrm{EE})>4 \mathrm{AB} \quad(4 \mathrm{EE})>1 \mathrm{AB} \quad$ (1EE); este orden también se observa en el nivel de abstracción y complejidad de los EE elaborados.

Las observaciones permitieron caracterizar el proceso para este grupo de estudiantes pero cabría preguntarse, sin embargo, si las características observadas resultan ser similares a la de otros grupos de estudiantes o si es propio de este proceso de explicitación. Por lo tanto, no se pretende generalizar los resultados hacia otros grupos y contextos, ya que se para ello amerita una ampliación y profundización de esta investigación.

En general, el proceso de construcción reflexiva de EE en el contexto de esta investigación presentó algunas características, que permiten describirlos como: (a) inicialmente confuso, (b) cognitivamente demandante de pensamiento abstracto, (c) reflexivo, (d) complejo, (e) sustancialmente creativo, (f) con diferenciación conceptual progresiva (g) conceptualmente discriminativo de enunciados como VM, EA y EC, y (h) explícitamente mejorable.

\section{Aspectos conceptuales de la ciencia considerados en la reflexión}

Los aspectos mencionados por los estudiantes sobre la ciencia al intentar construir los EE se muestran en la Tabla 7, independientemente de que estos se ubicaran como VM, EC y EA, ya que de alguna manera los estudiantes intentaron orientar sus esfuerzos hacia la expresión de ideas sobre la ciencia en torno a los conceptos que consideraron relevante en cada caso. Se puede observar que los estudiantes utilizaron un total de 18 aspectos conceptuales para expresar los diferentes enunciados: curiosidad, experiencia, imaginación, hechos, observación, resolución de problemas, experimentación, métodos, control, sistematicidad, conocimiento, verificación de conocimientos, invención, pensamiento analítico/crítico, explicación, dificultades y descubrimiento. 
Tabla 7. Frecuencia (F) de aspectos sobre la investigación científica considerados en los planteamientos elaborados por cada par de estudiantes en cada TPL

\begin{tabular}{|c|c|c|c|c|c|c|c|}
\hline \multirow{3}{*}{ ASPECTOS } & \multicolumn{5}{|c|}{$1 \mathrm{AB}$} & \multirow{3}{*}{$\begin{array}{l}\text { TP } \\
\text { L } 1\end{array}$} & \multirow{3}{*}{$\begin{array}{l}\text { TP } \\
\text { L } 2\end{array}$} \\
\hline & TP & TP & TP & TP & TP & & \\
\hline & L1 & L2 & L 3 & L 4 & L 5 & & \\
\hline
\end{tabular}

4.Hechos

5.Observación

6. Resol. problemas

7. Experimentación

8. Métodos

9. Control

10.Sistematicidad

11.Conocimiento

12.Verific conoc.

13.Invención

X X

X

14.Pensamiento

analítico/crítico

15.Explicación

16.Dificultades

17.Descubrimiento

18.Utilidad/beneficio

TP TP TPL TP TP TP

AB

$\begin{array}{llll}\text { TP } & \text { TP } & \text { TP } & \text { TP } \\ \text { L 4 } & \text { L 5 } & \text { L 1 } & \text { L 2 }\end{array}$

4AB

Variabilidad conceptual por

equipo

$\mathrm{X}$

X

X

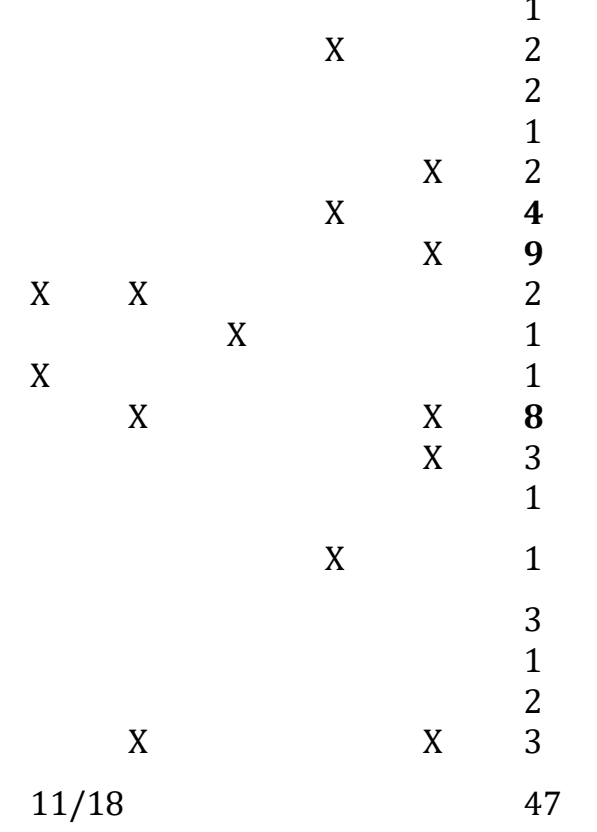

X X

X $\quad$ X

X

$\mathrm{X}$

$x$

X

$\mathrm{X}$

X

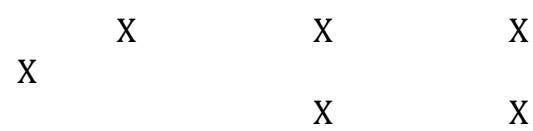

X

X

$\mathrm{X}$

\section{AB}

$\begin{array}{cccc}\text { TP } & \text { TPL } & \text { F } \\ \text { L } 4 & 5 & \end{array}$ 
Los resultados obtenidos por frecuencia de estos aspectos revelaron que tres de ellos exhibieron una mayor frecuencia de uso: experimentación (9/47), conocimiento (8/47) y resolución de problemas (4/47). Los aspectos referidos a invención, explicación y utilidad/beneficio recibieron el siguiente orden de atención por parte de los estudiantes para nuclear la construcción de sus enunciados (3/47). Si se toma en cuenta que el contexto de desarrollo de los TPL implicó experimentación para la búsqueda de la resolución de los problemas planteados en el laboratorio, se puede comprender la mayor frecuencia de los aspectos concernientes a la experimentación, conocimiento y resolución de problemas.

Sin embargo, la variedad conceptual utilizada por cada equipo de estudiantes puede constituir un criterio para establecer una diferencia en cuanto a la diversidad de ideas que emplearon al ser colocados en una situación de explicitación reflexiva de EE en el laboratorio; en este sentido, se puede decir que el siguiente orden de diversidad conceptual entre los equipos de estudiantes es válido en este estudio: $\mathbf{4 A B}$ (11) > 2AB (9) $>$ 1AB (6) > 3AB (5).como se aprecia en la Tabla 7. Los conceptos constituyen los elementos del pensamiento que permiten dinamizar las ideas, por lo que se pudiera entender que los estudiantes que emplearon una mayor diversidad conceptual disponen de una estructura cognoscitiva más compleja, tomando en consideración los planteamientos ausubelianos (Ausubel, 2002).
El conjunto de los 18 aspectos conceptuales expresados puede ser útil para organizar indagaciones más profundas y diseñar propuestas didácticas dinámicas $\mathrm{y}$ contextualizadas, orientadas hacia la modificación de ideas deformadas sobre la investigación científica o ciencia en general. Sin embargo, es importante tener presente que algunos de los EE elaborados no se pueden considerar conocimiento distal de manera purista, ya que pudieran entenderse como conocimiento proximal del estudiante (ver Tabla 3 para casos marcados con asteriscos); sin embargo, para fines de este trabajo se asumió como un EE igualmente válido, ya que se parte de la idea de que el pensamiento del estudiante estaba inmerso en su propia investigación como contexto pero trataba de reflexionar sobre la investigación científica profesional, lo cual es muy probable que esto ocurra en situaciones contextualizadas como la ocurrida en este estudio.

\section{Tendencias epistemológicas generales apreciables en los EE}

Por último, cabe señalar que los enunciados epistemológicos expresados por los estudiantes revelan cierta tendencia epistemológica, la cual se puede apreciar en los ejemplos representativos de la Tabla 8 tomando como referencia su tendencia contemporánea o tradicional, lo que implica una aproximación que amerita profundización investigativa. 
Tabla 8. Posibles tendencias epistemológicas sobre la ciencia de los estudiantes subyacentes en los enunciados construidos

\begin{tabular}{|c|c|c|}
\hline $\begin{array}{c}\text { PARES DE } \\
\text { ESTUDIANTES }\end{array}$ & ONES & $\begin{array}{l}\text { TENDENCIA } \\
\text { POSIBLE. }\end{array}$ \\
\hline
\end{tabular}

Estos estudiantes presentan inicialmente una visión poco clara de la naturaleza de la ciencia, ya que sus construcciones son en esencia creencias o conocimiento científico. Sin embargo, en los dos últimos TP, y aún en el tercero, se comienza a perfilar algo incipiente a un planteamiento filosófico donde se refleja el valor que le dan a los aspectos de la ciencia referidos a utilidad social, experimentación, curiosidad e invención. Es difícil, sin embargo, precisar una visión epistemológica definida de estos estudiantes en cuanto a la naturaleza de la ciencia. "Solucionar dificultades experimentales anima a inventar el mañana sin olvidar el justo valor del ayer".

Estos estudiantes presentan una visión objetiva de la naturaleza de la ciencia basada en la verificación del conocimiento, la experimentación y la resolución de problemas de la humanidad, así como en el papel del investigador, el cual es visto como un descubridor del mundo y no como constructor del mismo, siendo esto apreciable en el tercer y quinto TP. Sin embargo, en el cuarto Contemporánea TP se comienza a apreciar una visión más constructivista de la naturaleza de la ciencia, reflejada en la expresión: "la esencia del experimentador está en ver más allá de lo físicamente palpable". Estos estudiantes revelan inicialmente una visión objetiva de la naturaleza de la ciencia basada en el papel fundamental de la experimentación como en la verificación del conocimiento científico. Sin embargo, sus planteamientos reflejan que esta visión no es del todo objetivista, sino que tiende a lo constructivista, como se puede apreciar en las expresiones: "la imaginación es la piedra fundacional del experimentador" y "el conocimiento rompe las barreras de la mera experimentación". El conocimiento rompe las barreras de la mera experimentación".

Estos estudiantes reflejan una visión objetiva de la naturaleza de la ciencia sobre la base de la verificación del conocimiento, la experiencia, control de factores y utilidad social. No se aprecia un avance claro hacia una visión constructivista de la naturaleza de la Tradicional ciencia, como se aprecia en la expresión: "Los experimentos de No definible laboratorio, así como las observaciones, análisis y determinaciones... permiten confirmar y verificar los conocimientos...".

La ubicación realizada es muy general, por lo que convendría realizar investigaciones más detalladas al respecto, como la reportada por Flores et al. (2013a). Lo encontrado en esta experiencia exploratoria sirve de base para estructurar investigaciones de este tipo con una orientación y claridad mayor. 


\section{CONCLUSIONES}

Es importante destacar que el propósito de este trabajo no fue generar cambios en las concepciones deformadas de los estudiantes sino diagnosticarlas $y$ tener una aproximación comprensiva del proceso de construcción de enunciados epistemológicos sobre la ciencia en el contexto de los TPL desarrollados como resolución de problemas. De este modo, la realización de los TPL en el contexto didáctico de esta investigación sirvió de contexto para permitir la expresión consciente de los enunciados, los cuales revelaron un proceso complejo en el que emergieron cuatro tipos de enunciados, construidos con base en 18 conceptos sobre aspectos de la ciencia y con cierta tendencia epistemológica, constructivista en la mayoría de los casos.

En este sentido, las tendencias epistemológicas observadas no pueden ser atribuidas a la experiencia de los TPL con el diagrama $\mathrm{V}$, propiamente dicho, tomando en cuenta especialmente que no se desarrolló un estudio comparativo. Sin embargo, los resultados de este trabajo sirven de apoyo para diseñar estrategias didácticas que permitan mejorar las concepciones sobre la ciencia de los futuros docentes cursantes de Bioquímica en el IPC, sobre la base de las ideas deformadas encontradas y los resultados del proceso de explicitación reflexiva de los enunciados sobre ciencia, que dan respuesta a la pregunta de investigación planteada.

Para profundizar en investigaciones futuras, se recomienda: (a) orientar la construcción de enunciados epistemológicos hacia aspectos específicos de la investigación científica como la experimentación o los datos; (b) aplicar entrevistas semiestructuradas; (c) analizar la contribución del uso del diagrama $\mathrm{V}$ en la construcción de ideas epistemológicas apropiadas sobre la NDC; (d) teorizar el proceso de construcción de enunciados epistemológicos acerca de la ciencia; y (e) implementar un modelo didáctico que le permita a los estudiantes construir reflexivamente una concepción de la NDC más acorde con las tendencias filosóficas contemporáneas.

\section{REFERENCIAS}

Acevedo Díaz, J.A. (2008). «El estado actual de la naturaleza de la ciencia en la didáctica de las ciencias». Revista Eureka sobre Enseñanza y Divulgación de las Ciencias, 5(2), 134-169

Acevedo Díaz, J.A. (2009). «Enfoques explícitos versus implícitos en la enseñanza de la naturaleza de la ciencia». Revista Eureka sobre Enseñanza $y$ Divulgación de las Ciencias, 6(3), 356-386

Adúriz-Bravo, A. (2007). ¿Qué naturaleza de la ciencia hemos de saber los profesores de ciencias? Una cuestión actual de la investigación didáctica. UNESCO. Recuperado de http://es.scribd.com/doc /37819625 /Que-Naturaleza-de-LaCiencia-Hemos-de-Saber-Los-Profesores

Akerson, V.L., Abd-El-Khalik, F. y Lederman, N.G. (2000). «The influence of a reflective activity based approach on elementary teacher's conceptions of the nature of science». Journal of Research in Science Teaching, 37, 295-317

Ausubel, D.P. (2002). Adquisición y retención del conocimiento. Una perspectiva cognitiva. España: Editorial Paidós Ibérica, S.A

Barrera Morales, M.F. (2007). Análisis en investigación. Análisis semántico, de signos, significados y significaciones. Caracas: Quirón, S.A

Castañeda Figueiras, S. y Peñalosa Castro, E. (2010). «Validando constructos en epistemología personal»". Revista Mexicana de Psicología, 27(1), pp. 65-75. Recuperado de http://redalyc.uaemex.mx/ redalyc/pdf/2430/243016325007.pdf

Cobern, W. W. (2000). «The nature of science and the role of knowledge and belief». Science and Education, 9, 219-246

Elby, A. (2009). «Defining Personal epistemology: a response to Hofer \& 
Pintrich (1997) and Sandoval (2005) ». Journal of Learning Science, 18(1), 138149 . Recuperado de http://www2.physics.umd.edu/ elby/pa pers/Elby_reply_to_Sandoval_Hofer.pdf

Etkina, E., Murthy, S. y Zou, X. (2006). «Using introductory labs to engage students in experimental design». American Journal of Physics, 74(11), 979-986

Flores, J., Caballero, M.C. y Moreira, M.A. (2009). «El laboratorio en la enseñanza de la química: una visión integral en este complejo ambiente de aprendizaje». Revista de Investigación, 68(33), 75-111

Flores, J., Caballero, M.C. y Moreira, M.A. (2011). «Construcción de un marco teórico/conceptual para abordar el trabajo de laboratorio usando el diagrama V: un estudio de caso de la UPEL/IPC». Revista de Investigación, 73(35), 241-266

Flores, J., Caballero, M.C. y Moreira, M.A. (2013a). «Ideas epistemológicas sobre la naturaleza de la ciencia de docentes en formación de biología y de química». Qurriculum: Revista de Teoría, Investigación y Práctica Educativa, 26, 101-133

Flores, J., Caballero, M.C. y Moreira, M.A. (2013b). «Una interpretación de la teoría del aprendizaje significativo de Ausubel en el contexto del laboratorio didáctico de ciencias». Aprendizagem Significativa em Revista, 3(3), 41-54

Flores Espejo, J.L. (2014). «Un enfoque epistemológico-constructivista para facilitar el aprendizaje en el laboratorio desde una perspectiva ausubeliana». Tesis doctoral no publicada, Universidad de Burgos, España

Flores-Espejo, J.L. (2018). «Vivencia de aprendizaje sobre significados de naturaleza de la ciencia en un postgrado: mirada fenomenológica». Revista Gaceta de Pedagogía, 37, 191-224

Gowin, D. B. y Álvarez, M.C. (2005). The art of educating with $V$ diagrams. Cambridge: Cambridge University Press

Havdala, R. y Ashkenazi, G. (2007). «Coordination of theory and evidence: the effect of epistemological theories on studentes' laboratory practice». Journal of Research on Science Teaching, 44(8), 1134-1159

Hodson, D. (1994). «Hacia un enfoque más crítico del trabajo de laboratorio». Enseñanza de las Ciencias, 12(3), 299-313

Hogan, K. (2000). «Exploring a process view of student's knowledge about the nature of science». Science Education, 84, 51-70

Japiassu, H. (1991). Introducao ao pensamento epistemológico. Rio de Janeiro: Libraria

Lederman, N. (2006). «Research on nature of science: reflections on the past, anticipations of the future». Asia-Pacific Forum on Science Learning and Teaching, 7(1), 1-2. Recuperado de http://www.ied.edu.hk/apfslt/v7_issue1/ foreword/foreword2.htm\#two

Moreira, M.A. (2000). La teoría del aprendizaje significativo. En M.A.Moreira, C. Caballeros Sahelices y J. Meneses Villagrá (Orgs.), I Escuela de Verano sobre Investigación en Enseñanza de las Ciencias (pp. 211-251). España: Servicio de Publicaciones de la Universidad de Burgos

Moreira, M.A. (2006). Mapas conceituais \& diagramas $V$. Porto Alegre: Editorial do Autor

Moreira, M.A. y Levandowski, C.E. (1983). Diferentes abordagens ao ensino de laboratorio. Porto Alegre: Editora da Universidade

Novak, J.D. (1979). «Applying psychology and philosophy to improvement of laboratory teaching». The American Biology Teacher, 41(8), 466-474

Novak, J.D.y Gowin, B. (1988). Aprendiendo a aprender. España: Ediciones Martínez Roca

Orozco, R. A. (2019). «La ciencia y la filosofía: elementos de una educación integral». Recuperado https://www.redem.org/la-ciencia-y-lafilosofia-elementos-de-una-educacionintegral/

Sandoval, W, (2003). The inquiry paradox: why doing science doesn't necessarily change ideas about science. In C.P. 
Constantinou \& Zacharia (Eds.), Proceedings of the Sixth International Computer-Based Learning in Science Conference (pp. 825834). Nicosia, Cyprus
Séré, M. G. (2002). «Towards renewed research questions from the outcomes of the european project labwork in science education». Science Education, 86, 624-64 